\title{
LncRNA H19 contributes to hippocampal glial cell activation via JAK/STAT signaling in a rat model of temporal lobe epilepsy
}

Chun-Lei Han ${ }^{1,2}$, Ming Ge ${ }^{3}$, Yun-Peng Liu ${ }^{1,2}$, Xue-Min Zhao ${ }^{1,2}$, Kai-Liang Wang ${ }^{1,2}$, Ning Chen ${ }^{2,4}$, Wen-Jia Meng ${ }^{5}$, Wei $\mathrm{Hu}^{6}$, Jian-Guo Zhang ${ }^{2,4}$, Liang Li ${ }^{7^{*}}$ and Fan-Gang Meng ${ }^{1,2^{*}}$

\begin{abstract}
Background: Astrocyte and microglia activation are well-known features of temporal lobe epilepsy that may contribute to epileptogenesis. However, the mechanisms underlying glia activation are not well understood. Long non-coding RNA (IncRNA) H19 has diverse functions depending on physiological or pathological state, and its role in epilepsy is unknown. We previously demonstrated that $\mathrm{H} 19$ was significantly upregulated in the latent period of epilepsy and may be associated with cell proliferation and immune and inflammatory responses. We therefore speculated that $\mathrm{H} 19$ is involved in the hippocampal glial cell activation during epileptogenesis.
\end{abstract}

Methods: H19 was overexpressed or knocked down using an adeno-associated viral vector delivery system. A rat status epilepticus model was induced by intra-amygdala kainic acid injection. Astrocyte and microglia activation were assessed by immunofluorescence and western blot analyses. Expression of proinflammatory cytokines and components of the Janus kinase (JAK)/signal transducer and activator of transcription (STAT) signaling pathways were evaluated with western blotting.

Results: $\mathrm{H} 19$ overexpression induced the activation of astrocytes and microglia and the release of proinflammatory cytokines (interleukin- $1 \beta$ and interleukin- 6 and tumor necrosis factor- $\alpha$ ) in the hippocampus, whereas H19 knockdown inhibited status epilepticus-induced glial cell activation. Moreover, H19 activated JAK/STAT signaling by promoting the expression of Stat3 and c-Myc, which is thought to be involved in astrocyte activation.

Conclusions: LncRNA H19 contributes to hippocampal glial cell activation via modulation of the JAKISTAT pathway and could be a therapeutic tool to prevent the development of epilepsy.

Keywords: Temporal lobe epilepsy, IncRNA H19, Astrocytes, Microglia, Inflammatory response

\section{Background}

Temporal lobe epilepsy (TLE) is one of the most common types of intractable epilepsy and is characterized by the periodic and unpredictable occurrence of seizures. Glial cell activation and proliferation, a well-described pathological feature of TLE, can alter blood-brain barrier integrity and ion and neurotransmitter homeostasis and cause an inflammatory response, resulting in neuronal hyperexcitability and the generation and spread of

\footnotetext{
* Correspondence: liliang@ccmu.edu.cn; mengfg@ccmu.edu.cn

${ }^{7}$ Department of Pathology, School of Basic Medical Sciences, Capital Medical

University, No. 10 Xi TouTiao, You An Men Street, Beijing 100069, China

'Department of Functional Neurosurgery, Beijing Neurosurgical Institute,

Capital Medical University, Beijing 100050, China

Full list of author information is available at the end of the article
}

seizure activity [1-3]. Although impairment of these functions is thought to be associated with the pathophysiology of epilepsy, the mechanisms underlying glial cell activation are complex and are not fully understood [1].

Long non-coding RNA (lncRNA) H19, an imprinted gene, is located on human chromosome 11 and is transcribed from the maternally inherited allele [4]. Despite being identified over 20 years ago, the function of H19 remains unclear and its pathological role as a noncoding RNA has only recently been elucidated [5]. H19 has diverse functions depending on physiological and pathological state. In the central nervous system (CNS), H19 is overexpressed in glioblastoma tissue and promotes 
the proliferation, differentiation, migration, and invasion of glioma cells $[6,7]$. However, the biological function of H19 in non-neoplastic CNS diseases including epilepsy remains unknown.

We previously showed by high-throughput microarray and bioinformatics analyses that $\mathrm{H} 19$ is upregulated in the latent period of TLE in rat and is involved in various aspects of epileptogenesis, including cell proliferation and immune and inflammatory responses [8]. We therefore speculated that H19 may be involved in hippocampal glial cell activation during epileptogenesis. This was investigated in the present study by gain- and loss-offunction studies in a rat model of TLE. We also examined the possible downstream targets of H19.

\section{Methods}

\section{Animal and human samples}

Male Sprague-Dawley rats weighing 200-220 g were obtained from Vital River Experimental Animal Technology Co. (Beijing, China) and were housed in a temperaturecontrolled room with free access to standard food and water under a 12:12-h light/dark cycle. Surgically resected hippocampus specimens were obtained from patients with intractable TLE who underwent surgical treatment at Beijing Tiantan Hospital. Control hippocampal tissue was obtained from autopsies of four patients without a history of epilepsy or other neurological diseases within $8 \mathrm{~h}$ after death.

\section{H19 overexpression and knockdown}

H19 was overexpressed or silencing using an adenoassociated viral (AAV) vector delivery system as previously described [8]. Briefly, a vector harboring H19 (AAV-H19) or a short hairpin RNA targeting H19 (AAV-shRNA) was constructed by Gene Chem Co. (Shanghai, China). The negative control was an empty AAV vector (AAV-NC) or one harboring a scrambled sequence (AAV-Scr: 5'-TTCTCCGAACGTGTCACGT-3'). The titers used were $1.0 \times 10^{12}$ for AAV9-H19 and $4.0 \times$ $10^{12}$ for AAV9-shRNA. A total of $6 \mu \mathrm{l} \mathrm{AAV} \mathrm{was} \mathrm{infused}$ into the right dorsal hippocampus $(3.12 \mathrm{~mm}$ posterior to the bregma, $3.0 \mathrm{~mm}$ lateral to the midline, and 3 . $4 \mathrm{~mm}$ ventral to the bregma) and ventral hippocampus $(5.04 \mathrm{~mm}$ posterior to the bregma, $5.0 \mathrm{~mm}$ lateral to the midline, and $6.4 \mathrm{~mm}$ ventral to the bregma; $3 \mu \mathrm{l}$ at each location) [9] through a microsyringe at a speed of $0.2 \mu \mathrm{l} / \mathrm{min}$.

\section{Epilepsy model}

A kainic acid (KA)-induced status epilepticus (SE) model was established by intra-amygdala microinjection of KA 14 days after AAV injection according to our previously described technique [8]. Briefly, the rats were placed in a stereotaxic apparatus (David Kopf Instruments, Tujunga,
CA, USA), and $0.7 \mu \mathrm{l} \mathrm{KA}(1 \mu \mathrm{g} / \mu \mathrm{l}$; Sigma-Aldrich, St. Louis, MO, USA) was injected into the right amygdala ( $2.76 \mathrm{~mm}$ posterior to the bregma, $4.5 \mathrm{~mm}$ lateral to the midline, and $8.6 \mathrm{~mm}$ ventral to the bregma) [9] at a speed of $0.2 \mu \mathrm{l} / \mathrm{min}$. Sham-operated controls were injected with an equal volume of saline.

\section{Immunofluorescence analysis}

Coronal sections $(25 \mu \mathrm{m})$ were prepared at the level of the dorsal hippocampus $(2.50-3.50 \mathrm{~mm}$ posterior to the bregma). Frozen sections were dried, washed, permeabilized, blocked in 5\% goat serum, and incubated overnight with antibodies against neuronal nuclei (NeuN) (ab177487, 1:500 and ab104224, 1:200), glial fibrillary acidic protein (GFAP) (ab7260, 1:500), and OX42 (ab1211, 1:100) (all from Abcam, Cambridge, MA, USA). Immunolabeled sections were washed and incubated with goat secondary antibodies conjugated with Alexa Fluor 594 or Alexa Fluor 488 (Merck Biosciences, Nottingham, UK). Sections were mounted with medium containing 4',6-diamidino-2-phenylin-dole (DAPI) (Vector Laboratories, Burlingame, CA, USA), and images were captured using an inverted fluorescence microscope (Olympus, Tokyo, Japan). GFAP+ or OX42+ cells were manually counted using ImageJ software (US National Institutes of Health, Bethesda, MD, USA).

\section{Western blotting}

Western blot analysis was performed as previously described [10] using the following primary antibodies: rabbit polyclonal anti-GFAP (ab7260, 1:1000), mouse monoclonal anti-OX42 (ab1211, 1:500), rabbit polyclonal anti-interleukin (IL)-1 $\beta$ (ab9722; 1:500), mouse monoclonal anti-IL-6 (ab9324; 1:500), rabbit polyclonal anti-tumor necrosis factor (TNF)- $\alpha$ (ab6671; 1:500), rabbit monoclonal anti-p-Stat3 (ab76315; 1:500), and rabbit polyclonal anti-c-Myc (ab39688; 1:500) (all from Abcam, Cambridge, MA, USA). Rabbit monoclonal anti-glyceraldehyde 3phosphate dehydrogenase (GAPDH) antibody (Abcam, ab181602, 1:3000) was used as a control. Protein band density was quantified using an Epson V330 Photo scanner (Seiko Epson Co., Nagano, Japan) and Quantity One software (Bio-Rad, Hercules, CA, USA).

\section{Statistical analysis}

Data are presented as mean \pm standard error of the mean. Two-group comparisons were made with the unpaired Student's $t$ test, and multi-group comparisons were made by one-way analysis of variance followed by Sidak's multiple comparison tests using Prism 5 software (GraphPad Inc., San Diego, CA, USA). Significance was accepted at $P<0.05$. 


\section{Results}

Astrocytes and microglia are activated in the hippocampus of epileptic rats

We first examined GFAP and OX42 expression in the hippocampus of epileptic rats by immunofluorescence analysis to evaluate astrocyte and microglia activation, respectively. GFAP and OX42 immunoreactivity was detected in the ipsilateral hippocampus 7 days (latent period) (Figs. 2 and 3) or 30 days (chronic period) (Fig. 1) after KA-induced SE. The number of GFAP+ cells was increased, and activated astrocytes showed hypertrophy with a large cytoplasm and thick processes. The number of OX42+ cells was also increased, and the morphology of the activated microglia changed from spindle shape to oval with thickened processes. Fewer activated glial cells were observed in the contralateral as compared in the ipsilateral hippocampus (Fig. 1).

$\mathrm{H} 19$ is involved in the activation of astrocytes and microglia in the hippocampus of epileptic rats

Our previous study showed that H19 has diverse functions related to epileptogenesis [8] and is highly expressed in the seizure-free latent period of TLE. In the present study, we investigated the role of H19 in astrocyte and microglia activation by $\mathrm{H} 19$ overexpression and knockdown using an AAV delivery system [8]. Astrocyte and microglial activation were evaluated by GFAP and OX42 immunofluorescence and western blot analyses.
Compared to the sham group (NC + Veh or Scr + Veh), KA-induced SE (KA + Veh) or H19 overexpression (H19 + Veh) alone induced the activation of astrocytes or microglia in the stratum radiatum of the hippocampal CA3 region (Figs. 2 and 3). Moreover, more activated cells were observed in the hippocampus of rats overexpressing $\mathrm{H} 19$ at 7 days after SE (H19 + KA). The observed SE-induced activation of astrocytes and microglia was partly inhibited by H19 knockdown (ShRNA + KA vs Scr + KA). A quantitative analysis of GFAP and OX42 protein levels in the hippocampal CA3 region at 7 days (Figs. 2 and 3) and 60 days (Additional file 1: Figure S1) after SE confirmed these results.

We also examined proinflammatory cytokines released from the activated glia. H19 overexpression and KAinduced SE both stimulated the release of IL-1 $\beta$ and IL6 and TNF- $\alpha$ in the CA3 subfield of the hippocampus (Fig. 4a). Cytokine release was further increased by H19 at 7 days after SE (Fig. 4a). H19 knockdown prevented the SE-induced increase in IL- $1 \beta$ and IL- 6 and TNF- $\alpha$ levels (Fig. 4b). These results indicate that H19 plays an important role in astrocyte and microglia activation during epileptogenesis.

\section{H19 induces astrocyte and microglia activation via JAK/ STAT signaling}

Stat3 plays a key role in astrocyte proliferation after central nervous system injury $[11,12]$ or SE [13]. We found
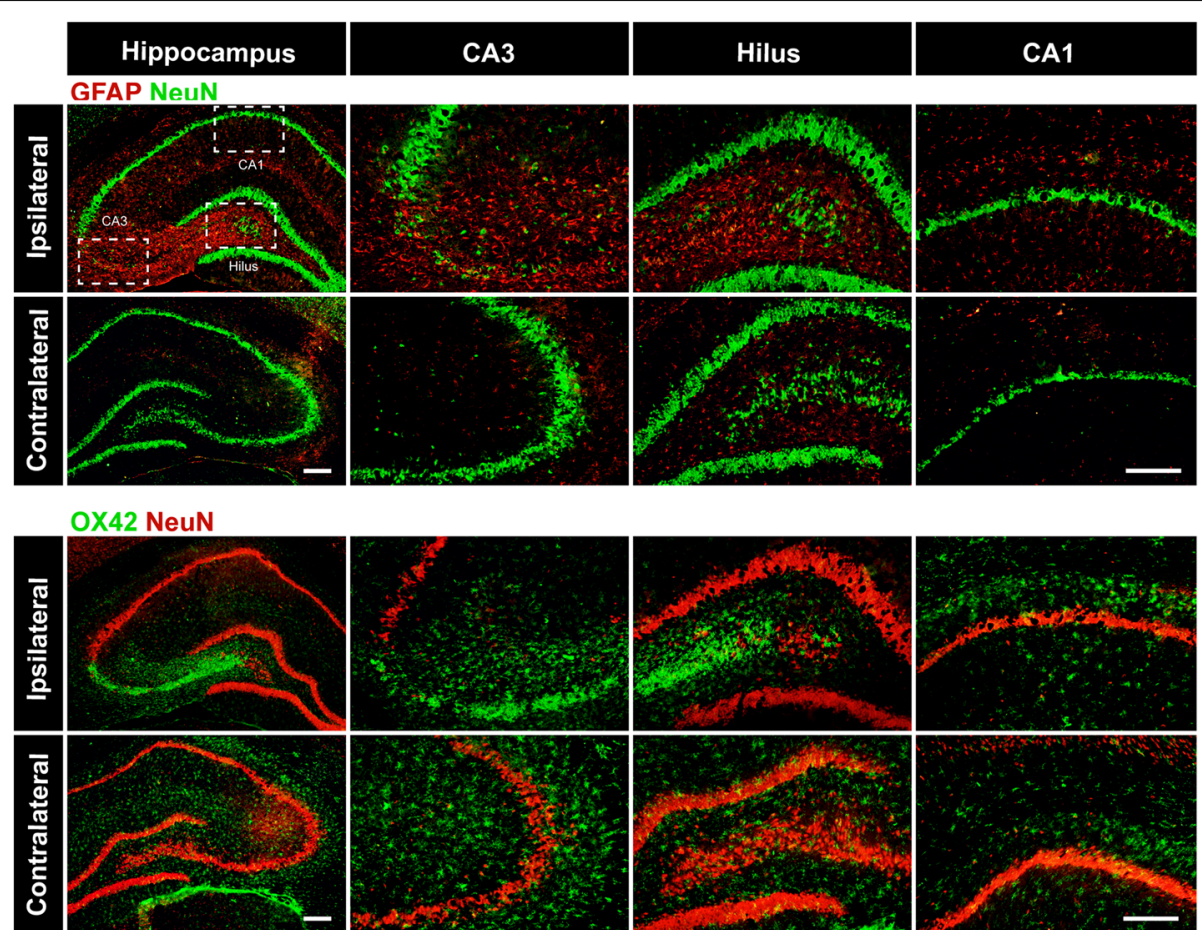

Fig. 1 Astrocytes and microglia are activated in the hippocampus of epileptic rats. Representative fluorescence micrographs of GFAP and OX42 expression in the hippocampus of rats 30 days after SE. High-magnification images correspond to the labeled boxes in the left panels. Scale bar = $200 \mu$ m 


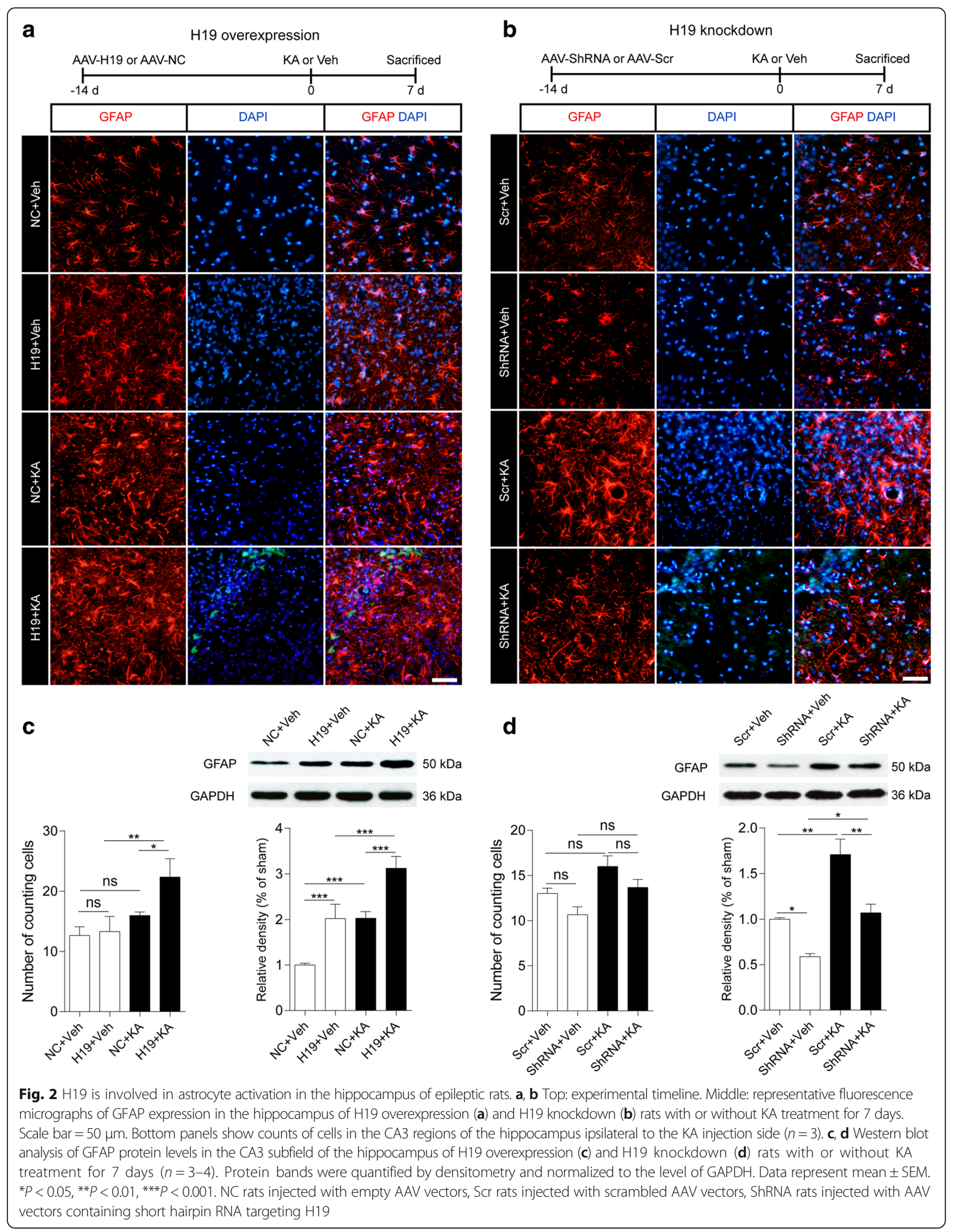




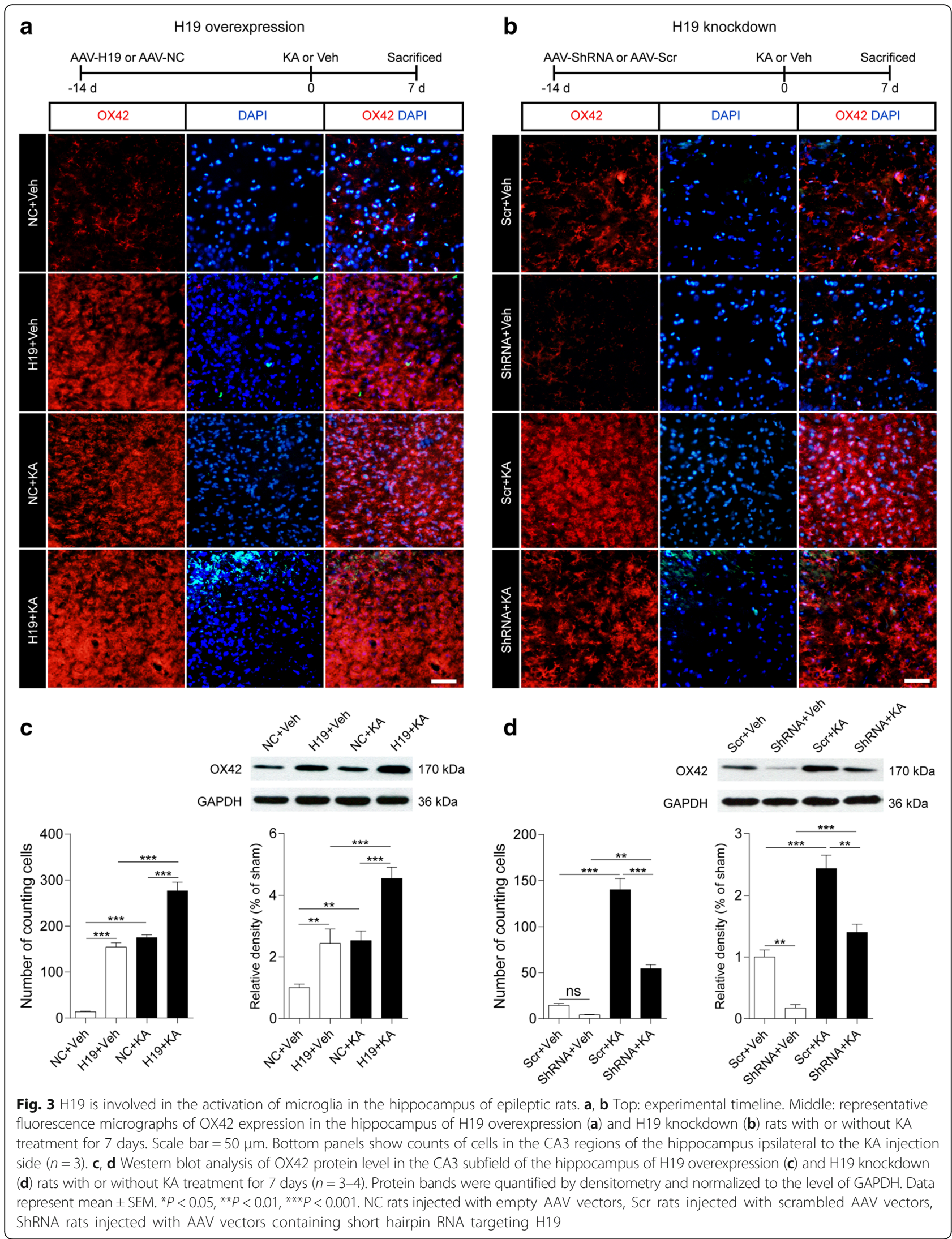




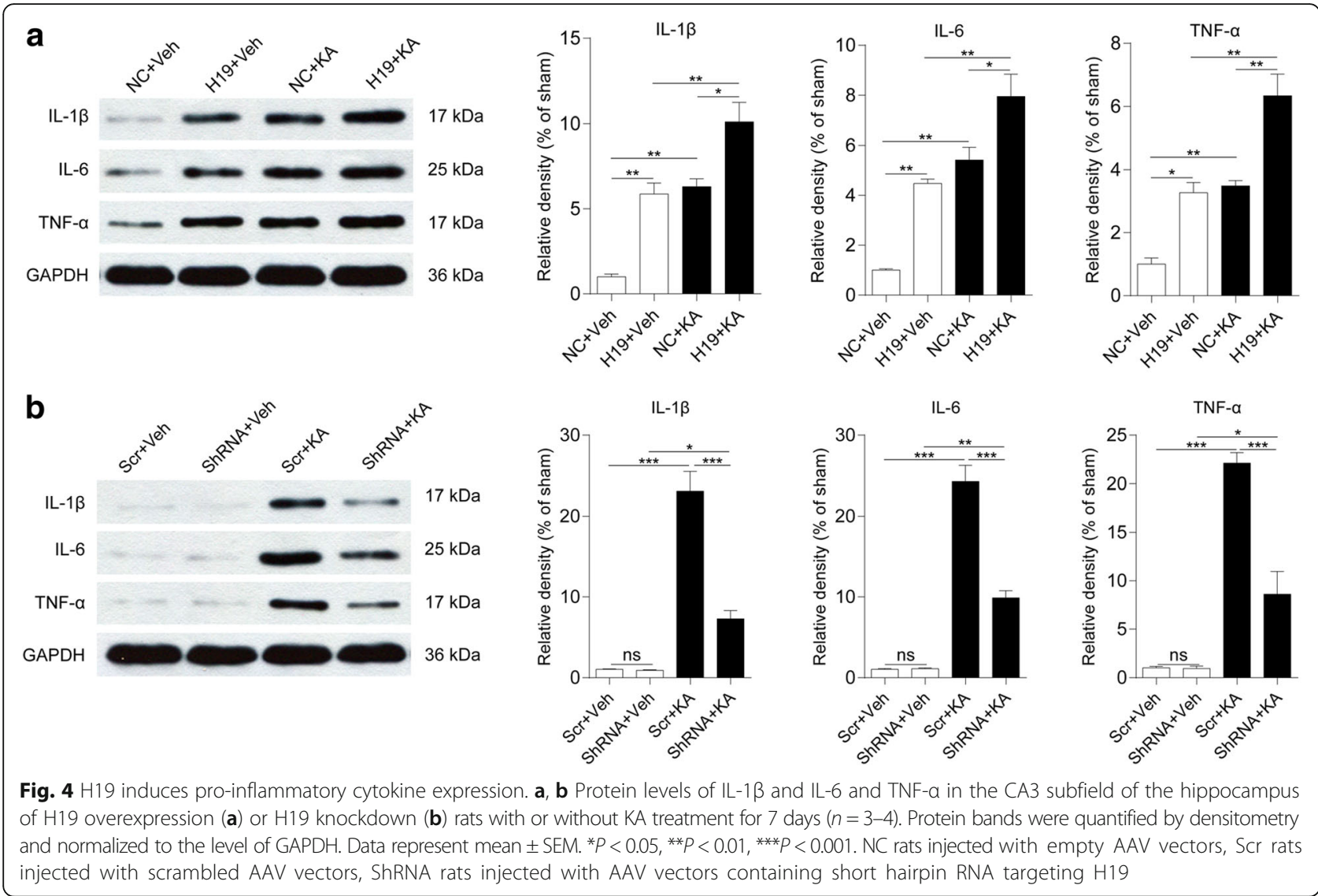

here that the levels of phosphorylated Stat3 (p-Stat3) and its downstream effector $\mathrm{c}-\mathrm{Myc}$ were upregulated in hippocampal tissue samples from patients with TLE (Fig. 5a) and from rats 7 days after SE (Fig. 5b) relative to the respective control samples, as determined with western blotting, suggesting that Janus kinase (JAK)/signal transducer and activator of transcription (STAT) signaling is involved in the activation of glial cells after SE. H19 overexpression alone increased p-Stat3 and c-Myc protein levels in the CA3 subfield of the hippocampus (Fig. 5c). H19 exacerbated these protein expressions in the rats at 7 days (Fig. 5c) and 60 days (Additional file 2: Figure S2A) after SE. On the contrary, H19 knockdown abolished the SEinduced increase in $\mathrm{p}$-Stat3 and $\mathrm{c}-\mathrm{Myc}$ in rats at 7 days (Fig. 5 d) and 60 days (Additional file 2: Figure S2B) after SE. These results indicate that $\mathrm{H} 19$ promotes astrocyte and microglial activation via the JAK/STAT signaling pathway.

\section{Discussion}

Most previous studies on H19 function focused on tumorigenesis. H19 was initially proposed as a tumor suppressor due to its capacity to suppress clonogenicity and tumorigenicity in tumor cells [14, 15]. However, recent studies showed that $\mathrm{H} 19$ acts as an oncogene by promoting cell proliferation, migration, invasion, and metastasis in various malignancies including glioblastoma [16-18]. Apart from these functions, H19 is also implicated in several other physiological conditions or diseases, such as cartilage degeneration in osteoarthritis [19], skeletal muscle differentiation and regeneration [20], and glucose metabolism in muscle cells [21]. However, the role of $\mathrm{H} 19$ in non-neoplastic CNS diseases including epilepsy remains unclear. In the present study, we provide the first evidence that H19 promotes glial cell activation and stimulates inflammation in the hippocampus of rats with TLE.

Astrocyte activation is a continuum that includes changes in molecular expression, progressive cellular hypertrophy, and, in severe cases, proliferation and scar formation [22]. In mild or moderate astrocyte activation, GFAP expression is slightly upregulated and the cell body and processes undergo hypertrophy, with little or no astrocyte proliferation. However, in severe diffuse reactive astrogliosis, GFAP expression is markedly increased, which is accompanied by extensive hypertrophy of the cell body and processes and astrocyte proliferation [23]. In the present study, astrocytes in the hippocampus of epileptic rats were activated in the latent and chronic 


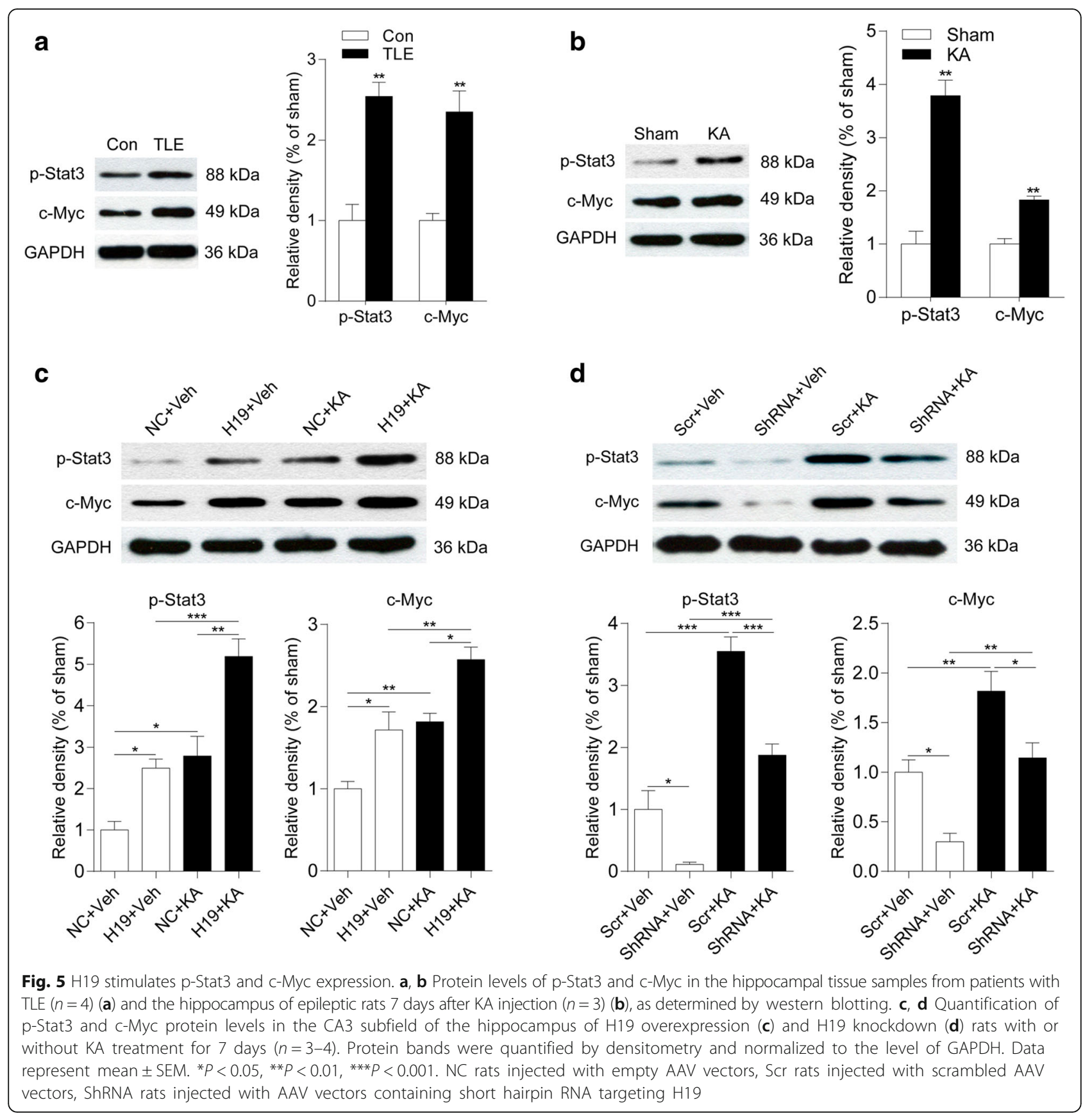

phases of TLE, which is consistent with previous reports $[13,24]$. Mild to moderate gliosis, which typically does not cause astrocyte proliferation, is usually observed in the early stages after SE [25]. We also found that astrocyte activation was mild or moderate, as evidenced by the upregulation of GFAP expression and cellular hypertrophy without an obvious increase in cell number in the latent period of TLE (7 days after SE). Furthermore, H19 induced an increase in GFAP expression and hypertrophy of astrocytes rather than cell proliferation in the latent period of SE. In the chronic period (30 days after
$\mathrm{SE}$ ), astrocyte activation was extreme with severe hypertrophy of the cell body and processes; moreover, astrocyte proliferation in areas of pyramidal neuron loss was comparable to the hippocampal sclerosis observed in TLE patients [26]. Unlike astrocytes, high expression of OX42 protein and cellular hypertrophy as well as proliferation of microglia was observed in both the latent and chronic periods of TLE, as previously reported [27]. Furthermore, H19 induced the upregulation of OX42 and cellular hypertrophy and increased the number of microglia in the latent period of SE. Recent studies have 
shown that inflammatory cytokines are produced both by microglia and astrocytes [28]; the increased levels of proinflammatory cytokines in epileptic rats observed here is in agreement with these findings. Moreover, H19 stimulated the release of proinflammatory cytokines. However, compared to sham rats, H19 knockdown did not inhibit proinflammatory cytokine expression, possibly because under normal conditions, proinflammatory cytokine levels in the hippocampus are too low to result in an observable difference upon H19 knockdown.

Molecular triggers that lead to glial cell activation and proliferation have not been fully characterized. There is increasing evidence to suggest that $\mathrm{H} 19$ has a growthpromoting function, since it enhances cell proliferation in tumors $[29,30]$ and other diseases $[20,31]$. In the present study, we showed that $\mathrm{H} 19$ promotes astrocyte and microglia activation and proliferation under both epileptic and normal conditions. This is consistent with earlier reports as well as with our previous research [8]. Various intracellular signaling pathways associated with Stat3, nuclear factor $\mathrm{\kappa B}$, and nuclear respiratory factor (Nrf) mediate cell hypertrophy, proliferation, and proor anti-inflammatory effects in astrocytes [23]. The transcription factor Stat3, a key component of the JAK/ STAT pathway, is important for astrocyte proliferation in CNS diseases [11, 32, 33]. P-Stat3 is highly expressed in the rat hippocampus during different phases of epilepsy and in the temporal lobe of TLE patients [13]. Astrocyte activation can be suppressed by inhibiting JAK/STAT signaling, indicating that Stat3 activation induces GFAP expression [13]. In our study, Stat3 as well as its downstream effector c-Myc in the JAK/STAT pathway were upregulated after SE in the rat hippocampus and in TLE patients. Furthermore, H19 overexpression induced whereas its knockdown inhibited Stat3 and c-Myc protein expression in both normal and epileptic rats. Thus, H19 can itself promote gliosis via JAK/STAT signaling in addition to its role in astrocyte activation.

\section{Conclusions}

In summary, lncRNA H19 contributes to the activation of hippocampal astrocytes and microglia, as well as to the inflammatory response in epileptic rats. Furthermore, H19 may promote glial cell activation through the JAK/STAT pathway. Our findings reveal a novel lncRNA H19-mediated mechanism in seizure-induced glial cell activation and provide a basis for developing IncRNAbased strategies to prevent the development of epilepsy.

\section{Additional files}

Additional file 1: H19 promotes GFAP and OX42 expression. (a, b) Western blot analysis of GFAP and OX42 protein levels in the CA3 subfield of the hippocampus of H19 overexpression (a) or H19 knockdown (b) rats with or without KA treatment for 60 days $(n=3-4)$. Protein bands were quantified by densitometry and normalized to GAPDH level. Data represent mean \pm SEM. ${ }^{*} P<0.05,{ }^{* *} P<0.01,{ }^{* * *} P<0.001$. NC, rats injected with empty AAV vectors; Scr, rats injected with scrambled AAV vectors; ShRNA, rats injected with AAV vectors containing short hairpin RNA targeting H19. (TIFF 469 kb)

Additional file 2: $\mathrm{H} 19$ promotes $\mathrm{p}-\mathrm{Stat} 3$ and c-Myc expression. (a, b) Western blot analysis of p-Stat3 and c-Myc protein levels in the CA3 subfield of hippocampus of H19 overexpression (a) or H19 knockdown (b) rats with or without KA treatment for 60 days $(n=3-4)$. Protein bands were quantified by densitometry and normalized to GAPDH level. Data represent mean \pm SEM. ${ }^{*} P<0.05,{ }^{* *} P<0.01$, ${ }^{* *} P<0.001$. NC, rats injected with empty AAV vectors; Scr, rats injected with scrambled AAV vectors; ShRNA, rats injected with AAV vectors containing short hairpin RNA targeting H19. (TIFF $454 \mathrm{~kb}$ )

\section{Abbreviations}

AAV: Adeno-associated viral; CNS: Central nervous system; DAPI: 4',6-diamidino2-phenylin-dole; GAPDH: Glyceraldehyde 3-phosphate dehydrogenase; GFAP: Glial fibrillary acidic protein; IL: Interleukin; JAK: Janus kinase; KA: Kainic acid; IncRNA: Long non-coding RNA; NC: Negative control; NeuN: Neuronal nuclei; Nrf: Nuclear respiratory factor; p-Stat3: Phosphorylated Stat3; SE: Status epilepticus; shRNA: Short hairpin RNA; STAT: Signal transducer and activator of transcription; TLE: Temporal lobe epilepsy; TNF: Tumor necrosis factor

\section{Acknowledgements}

Not applicable

\section{Funding}

This study was supported by the National Natural Science Foundation of China (81471315).

\section{Availability of data and materials}

All data are provided in the manuscript and in the additional files.

\section{Authors' contributions}

CLH performed the experiments and drafted the manuscript. MG analyzed the data. YPL and XMZ established the epilepsy model. KLW performed the intro-hippocampus AAV vector injection. NC performed the immunofluorescence image analysis. WH contributed to the experimental design. WJM performed the animal experiment of AG 490. JGZ assisted with the experiment design and collected the hippocampal samples from patients with TLE. LL designed the study and revised the manuscript. FGM designed and guided the experiment. All authors have read and approved the final version of the manuscript

\section{Ethics approval and consent to participate}

All experimental protocols involving animals were in compliance with the Chinese Animal Welfare Act and Beijing Guidelines for the Care and Use of Laboratory Animals. Written informed consent was obtained from each patient for the use of brain tissue for research purposes. The protocol was approved by the Ethics Committee of Beijing Neurosurgical Institute, Capital Medical University (process no. 201402019).

\section{Consent for publication}

Not applicable

\section{Competing interests}

The authors declare that they have no competing interests.

\section{Publisher's Note}

Springer Nature remains neutral with regard to jurisdictional claims in published maps and institutional affiliations.

\section{Author details}

${ }^{1}$ Department of Functional Neurosurgery, Beijing Neurosurgical Institute, Capital Medical University, Beijing 100050, China. ${ }^{2}$ Beijing Key Laboratory of Neuromodulation, Beijing Municipal Science and Technology Commission, Beijing 100050, China. 'Department of Neurosurgery, Beijing Children's 
Hospital, Capital Medical University, Beijing 100045, China. ${ }^{4}$ Department of Neurosurgery, Beijing Tiantan Hospital, Capital Medical University, Beijing 100050, China. ${ }^{5}$ The Third Division of Clinical Medicine, China Medical University, Shenyang 110122, Liaoning Province, China. ${ }^{6}$ Department of Neurology, University of Florida, Gainesville, Florida 32607, USA. Department of Pathology, School of Basic Medical Sciences, Capital Medical University, No. 10 Xi TouTiao, You An Men Street, Beijing 100069, China.

\section{Received: 15 December 2017 Accepted: 27 March 2018}

Published online: 10 April 2018

\section{References}

1. Seifert G, Carmignoto G, Steinhauser C. Astrocyte dysfunction in epilepsy. Brain Res Rev. 2010;63:212-21.

2. Perea G, Navarrete M, Araque A. Tripartite synapses: astrocytes process and control synaptic information. Trends Neurosci. 2009;32:421-31.

3. Halassa MM, Haydon PG. Integrated brain circuits: astrocytic networks modulate neuronal activity and behavior. Annu Rev Physiol. 2010;72:335.

4. Pachnis V, Belayew A, Tilghman SM. Locus unlinked to alpha-fetoprotein under the control of the murine raf and Rif genes. Proc Natl Acad Sci U S A. 1984;81:5523-7.

5. Liang WC, Fu WM, Wong CW, Wang Y, Wang WM, Hu GX, Zhang L, Xiao LJ, Wan DC, Zhang JF, Waye MM. The IncRNA H19 promotes epithelial to mesenchymal transition by functioning as miRNA sponges in colorectal cancer. Oncotarget. 2015;6:22513-25.

6. Jiang X, Yan Y, Hu M, Chen X, Wang Y, Dai Y, Wu D, Wang Y, Zhuang Z, Xia H. Increased level of $\mathrm{H} 19$ long noncoding RNA promotes invasion, angiogenesis, and stemness of glioblastoma cells. J Neurosurg. 2016;124:129-36.

7. Jia P, Cai H, Liu X, Chen J, Ma J, Wang P, Liu Y, Zheng J, Xue Y. Long noncoding RNA H19 regulates glioma angiogenesis and the biological behavior of glioma-associated endothelial cells by inhibiting microRNA-29a. Cancer Lett. 2016;381:359-69.

8. Han CL, Liu YP, Zhao XM, Wang KL, Chen N, Hu W, Zhang JG, Ge M, Meng FG. Whole-transcriptome screening reveals the regulatory targets and functions of long non-coding RNA H19 in epileptic rats. Biochem Biophys Res Commun. 2017:489:262-9.

9. Paxinos G, Watson C. The rat brain in stereotaxic coordinates. 5th ed. San Diego: Elsevier Academic Press; 2005.

10. Wu X, Sun J, Zhang X, Li X, Liu Z, Yang Q, Li L. Epigenetic signature of chronic cerebral hypoperfusion and beneficial effects of S-adenosylmethionine in rats. Mol Neurobiol. 2014:50:839-51.

11. Herrmann JE, Imura T, Song B, Qi J, Ao Y, Nguyen TK, Korsak RA, Takeda K, Akira S, Sofroniew MV. STAT3 is a critical regulator of astrogliosis and scar formation after spinal cord injury. J Neurosci. 2008;28:7231-43.

12. Tsuda M, Kohro Y, Yano T, Tsujikawa T, Kitano J, Tozaki-Saitoh H, Koyanagi S, Ohdo S, Ji RR, Salter MW, Inoue K. JAK-STAT3 pathway regulates spinal astrocyte proliferation and neuropathic pain maintenance in rats. Brain. 2011;134:1127-39

13. $X u Z$, Xue T, Zhang Z, Wang $X, X u P$, Zhang J, Lei $X$, Li Y, Xie Y, Wang L, et al. Role of signal transducer and activator of transcription-3 in up-regulation of GFAP after epilepsy. Neurochem Res. 2011;36:2208-15.

14. Hao Y, Crenshaw T, Moulton T, Newcomb E, Tycko B. Tumour-suppressor activity of H19 RNA. Nature. 1993;365:764-7.

15. Yoshimizu T, Miroglio A, Ripoche MA, Gabory A, Vernucci M, Riccio A, Colnot S, Godard C, Terris B, Jammes H, Dandolo L. The H19 locus acts in vivo as a tumor suppressor. Proc Natl Acad Sci U S A. 2008;105:12417-22.

16. Yan L, Zhou J, Gao Y, Ghazal S, Lu L, Bellone S, Yang Y, Liu N, Zhao X, Santin $A D$, et al. Regulation of tumor cell migration and invasion by the H19/let-7 axis is antagonized by metformin-induced DNA methylation. Oncogene. 2015;34:3076-84.

17. Shi Y, Wang Y, Luan W, Wang P, Tao T, Zhang J, Qian J, Liu N, You Y. Long non-coding RNA H19 promotes glioma cell invasion by deriving miR-675. PLoS One. 2014;9:e86295.

18. Matouk IJ, DeGroot N, Mezan S, Ayesh S, Abu-lail R, Hochberg A, Galun E. The H19 non-coding RNA is essential for human tumor growth. PLoS One. 2007;2:e845

19. Steck E, Boeuf S, Gabler J, Werth N, Schnatzer P, Diederichs S, Richter W. Regulation of $\mathrm{H} 19$ and its encoded microRNA-675 in osteoarthritis and under anabolic and catabolic in vitro conditions. J Mol Med (Berl). 2012;90: 1185-95.
20. Dey BK, Pfeifer K, Dutta A. The H19 long noncoding RNA gives rise to microRNAs miR-675-3p and miR-675-5p to promote skeletal muscle differentiation and regeneration. Genes Dev. 2014;28:491-501.

21. Gao Y, Wu F, Zhou J, Yan L, Jurczak MJ, Lee HY, Yang L, Mueller M, Zhou XB, Dandolo L, et al. The H19/let-7 double-negative feedback loop contributes to glucose metabolism in muscle cells. Nucleic Acids Res. 2014:42:13799-811.

22. Sofroniew MV, Vinters HV. Astrocytes: biology and pathology. Acta Neuropathol. 2010;119:7-35.

23. Sofroniew MV. Molecular dissection of reactive astrogliosis and glial scar formation. Trends Neurosci. 2009;32:638-47.

24. Shapiro LA, Wang L, Ribak CE. Rapid astrocyte and microglial activation following pilocarpine-induced seizures in rats. Epilepsia. 2008;49(Suppl 2): 33-41.

25. Gibbons MB, Smeal RM, Takahashi DK, Vargas JR, Wilcox KS. Contributions of astrocytes to epileptogenesis following status epilepticus: opportunities for preventive therapy? Neurochem Int. 2013;63:660-9.

26. Thom M. Review: hippocampal sclerosis in epilepsy: a neuropathology review. Neuropathol Appl Neurobiol. 2014;40:520-43

27. Avignone E, Ulmann L, Levavasseur F, Rassendren F, Audinat E. Status epilepticus induces a particular microglial activation state characterized by enhanced purinergic signaling. J Neurosci. 2008;28:9133-44.

28. Rizzi M, Perego C, Aliprandi M, Richichi C, Ravizza T, Colella D, Veliskova J, Moshe SL, De Simoni MG, Vezzani A. Glia activation and cytokine increase in rat hippocampus by kainic acid-induced status epilepticus during postnatal development. Neurobiol Dis. 2003;14:494-503.

29. Tan D, Wu Y, Hu L, He P, Xiong G, Bai Y, Yang K. Long noncoding RNA H19 is up-regulated in esophageal squamous cell carcinoma and promotes cell proliferation and metastasis. Dis Esophagus. 2017;30:1-9.

30. lempridee T. Long non-coding RNA H19 enhances cell proliferation and anchorage-independent growth of cervical cancer cell lines. Exp Biol Med (Maywood). 2017;242:184-93.

31. Xu X, Ji S, Li W, Yi B, Li H, Zhang H, Ma W. LncRNA H19 promotes the differentiation of bovine skeletal muscle satellite cells by suppressing Sirt1/FoxO1. Cell Mol Biol Lett. 2017;22:10.

32. Satriotomo I, Bowen KK, Vemuganti R. JAK2 and STAT3 activation contributes to neuronal damage following transient focal cerebral ischemia. J Neurochem. 2006;98:1353-68.

33. Ben Haim L, Ceyzeriat K, Carrillo-de Sauvage MA, Aubry F, Auregan G, Guillermier M, Ruiz M, Petit F, Houitte D, Faivre E, et al. The JAK/STAT3 pathway is a common inducer of astrocyte reactivity in Alzheimer's and Huntington's diseases. J Neurosci. 2015;35:2817-29.

\section{Submit your next manuscript to BioMed Central and we will help you at every step:}

- We accept pre-submission inquiries

- Our selector tool helps you to find the most relevant journal

- We provide round the clock customer support

- Convenient online submission

- Thorough peer review

- Inclusion in PubMed and all major indexing services

- Maximum visibility for your research

Submit your manuscript at www.biomedcentral.com/submit
) Biomed Central 